BULLETIN OF THE

AMERICAN MATHEMATICAL SOCIETY

Volume 80, Number 3, May 1974

\title{
SMOOTH MAPS OF CONSTANT RANK
}

\author{
BY ANTHONY PHILLIPS
}

Communicated by William Browder, July 10, 1973

1. Introduction. In this announcement the Smale-Hirsch classification of immersions ([8], [5]) is extended to maps of arbitrary constant rank, under certain conditions on the source manifold.

THEOREM 1. If $M$ is open and has a proper Morse function with no critical points of index $>k$, then the differential map $d: \operatorname{Hom}_{k}(M, W) \rightarrow$ $\operatorname{Lin}_{k}(T M, T W)$ is a weak homotopy equivalence.

(A manifold with such a Morse function will be said to have geometric dimension $\leqq k$. We will write geo $\operatorname{dim} M \leqq K$.)

Notation. $M$ and $W$ are smooth manifolds with tangent bundles $T M$, $T W ; \operatorname{Hom}_{k}(M, W)$ is the space of smooth maps of rank $k$ from $M$ to $W$, with the $C^{1}$-compact-open topology; $\operatorname{Lin}_{k}(T M, T W)$ is the space of continuous maps: $T M \rightarrow T W$ which are fiberwise linear maps of rank $k$, with the compact open topology; $d(f)=d f$.

Remarks. 1. Weakening the hypotheses leads to false statements. If $M$ is not open there are counterexamples when $k=\operatorname{dim} W$ as in [7]. Otherwise, take $M$ to be the parallelizable manifold $S^{k+1} \times R$; then the identity map of $M$ can be covered by $H \in \operatorname{Lin}_{k}(T M, T M)$ but $H$ cannot be homotopic to the differential of an $f \in \operatorname{Hom}_{k}(M, M)$ since such an $f$ (by Sard's theorem) would be null-homotopic. I owe this example to David Frank.

2. When $k=\operatorname{dim} M$ this gives the Smale-Hirsch theorem for open manifolds, but when $k=\operatorname{dim} W$ this does not give the full classification of submersions [7]. The missing cases will be considered in a future article. (ADDED IN PROOF. A necessary and sufficient condition for $H \in$ $\operatorname{Lin}_{k}(T M, T W)$ to be homotopic to the differential of some $f \in \operatorname{Hom}_{k}(M, W)$ is given, for arbitrary open $M$, in M. L. Gromov, Singular smooth maps, Mat. Zametki 14 (1973), 509-516. It is equivalent to requiring that $H$ factor through a $k$-dimensional bundle over a $k$-dimensional complex.) Immersions and submersions are the only overlap between this theorem and Feit's classification of $k$-mersions (maps of rank everywhere $\geqq k$ ) [2]

3. This theorem is not a special case of Gromov's theorem [3], since

AMS (MOS) subject classifications (1970). Primary 57D30, 57D35; Secondary 55F65, 57D40, 57D45, 58A30, 58C25, 58D10.

Copyright (c) American Mathematical Society 1974 
having rank $k$ is not an open condition in general. We will, however, make heavy use of the ideas and results of [3] throughout this work.

4. A map of constant rank is locally a submersion followed by an immersion, i.e., a subimmersion. The ensuing local "stability" is the key to our proof. It also follows that inverse images of points under a map of constant rank foliate the source manifold. An application of Theorem 1 is then this weak form of a theorem of [4]. On an open manifold $M$ of geometric dimension $\leqq k$, any plane field $\sigma$ of codimension $k$ is homotopic to an integrable field. The proof is immediate, since projection onto the complementary bundle $\sigma^{\perp}$ can be considered as a bundle map of rank $k$ from $T M$ to the tangent bundle of the total space of $\sigma^{\perp}$.

The proof of Theorem 1 has two main steps: First, the manifold $M$ is assumed to be highly coconnected; then the general case is reduced to this special one.

2. Proof for highly coconnected manifolds. Let $a(0)=a(1)=0, a(2)=$ $a(3)=1$, and $a(x)=\frac{1}{2}(x-1)$ if $x \geqq 4$.

THEOREM 1'. Let $\operatorname{dim} M=n$. If $\operatorname{geo} \operatorname{dim} M \leqq \min (a(n), k)$, then $d$ : $\operatorname{Hom}_{k}(M, W) \rightarrow \operatorname{Lin}_{k}(T M, T W)$ is a weak homotopy equivalence.

The theorem proving machine ([3], [6]) reduces the proof to showing that the restriction map $\operatorname{Hom}_{k}(V, W) \rightarrow \operatorname{Hom}_{k}(U, W)$ has the covering homotopy property, when $U \subset V \subset M$ are $n$-dimensional submanifolds, and $V=U \cup$ handle of index $\lambda \leqq \min (a(n), k)$. This is not true in general (see Figure 1), but, as pointed out to me by Edgar Feldman, the weak covering homotopy property [1] is sufficient (this allows a preliminary vertical homotopy; see below). Using 3.2.3 of [3] ( $r$-microflexible implies $r$-flexible) we can further reduce our problem to the following lemma.

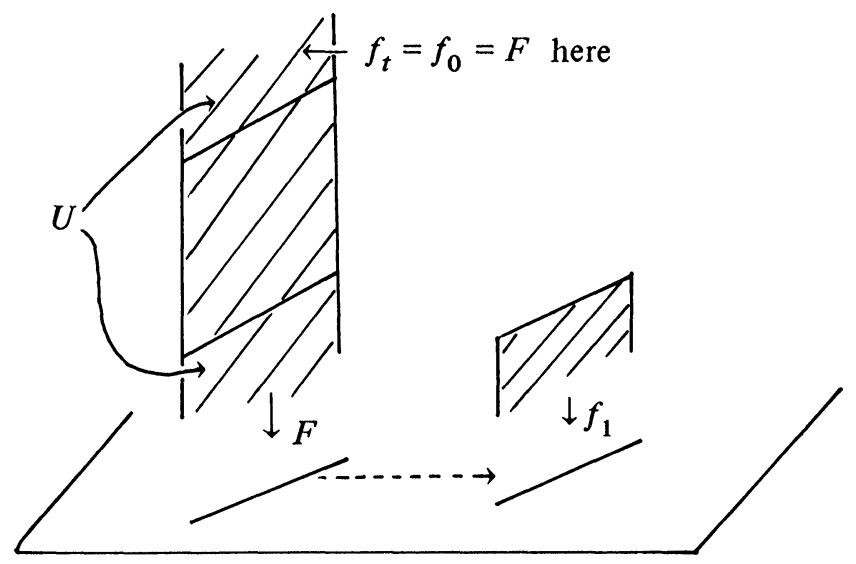

FIGURE 1 
Weak Micro-Covering Homotopy Lemma. Suppose we are given $U, V$ as above, a compact $P$ and continuous maps $F: P \rightarrow \operatorname{Hom}_{k}(V, W)$ and $f: P \times[0,1] \rightarrow \operatorname{Hom}_{k}(U, W)$ with $f_{p, 0}=F_{p} \mid U$ for $p \in P$. Then there exist $\varepsilon>0$ and a continuous $\widetilde{F}: P \times[-1, \varepsilon] \rightarrow \operatorname{Hom}_{k}(V, W)$ with $\widetilde{F}_{p,-1}=$ $F_{p}$ for $p \in P$, such that $\tilde{F}_{p, t} \mid U=f_{p, 0}$ if $t \leqq 0$ and $=f_{p, t}$ if $0 \leqq t \leqq \varepsilon$, for $p \in P$.

SKeTCH OF PROOF. So as not to obscure the geometry, I will take $P=$ a point and leave it out of the notation. We then consider $F \in \operatorname{Hom}_{k}(V, W)$ and a homotopy $f: I \rightarrow \operatorname{Hom}_{k}(U, W)$ with $f_{0}=F \mid U$.

Let us admit ${ }^{1}$ that the homotopy $f_{t}$ is defined on a "collar neighborhood" $N$ (as in [7]) of $U$ in $V$. By Remark 4 above, there exists a disc $D^{n}$ about any point of $N$ such that $f_{t} \mid D^{n}$ is a subimmersion, for $0 \leqq t \leqq 1$. This suffices for the case $k=\lambda=1$ (everything is trivial when $k=0$ ): we construct an isotopy of $N-U$ in itself which deforms the identity to a map pulling each component of $N-U$ through such a $D^{n}$, across the foliation defined there by $F$ (see Figure 2). Then after a preliminary homotopy defined by com-

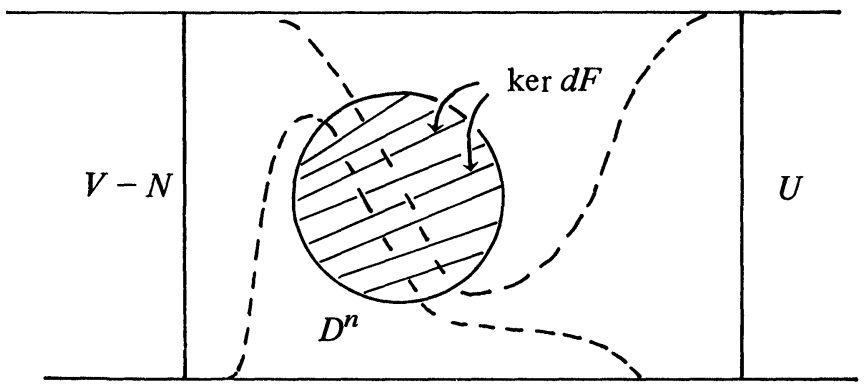

FIGURE 2

posing $F$ with this isotopy, the stability of submersions and immersions can be used to give an initial lifting, as required. The deformation corresponding to the problem of Figure 1 might be as in Figure 3.

In general $N-U \cong S^{\lambda-1} \times D^{n-\lambda} \times I$. The subset corresponding to the two discs would be a tubular neighborhood of the "core" $S=S^{\lambda-1} \times$ $\{0\} \times\left\{\frac{1}{2}\right\}$. If $F \mid S$ is an immersion, then $F$ subimmerses a tubular neighborhood $T$ of $S$, and we proceed as before, using a preliminary isotopy which draws $N-U$ through $T$, across the foliation defined in $T$ by $F$.

It is clearly sufficient to show that $S$ is isotopic to a sphere immersed by $F$; this is proved in three steps. First, using 5.2.1 of [3] and the hypothesis $\lambda \leqq k$, the inclusion $i: S \rightarrow N-U$ is homotopic to an immersion $i^{\prime}$

\footnotetext{
${ }^{1}$ This leap of faith is not required if, for $U \subset M, \operatorname{Hom}_{k}(U, W)$ is defined as inj $\lim \operatorname{Hom}_{k}(A, W)$, where $A$ runs through the family of open neighborhoods of $U$ in $M$, and is given the quasi-topology it inherits as inj lim. See [3, \$2].
} 


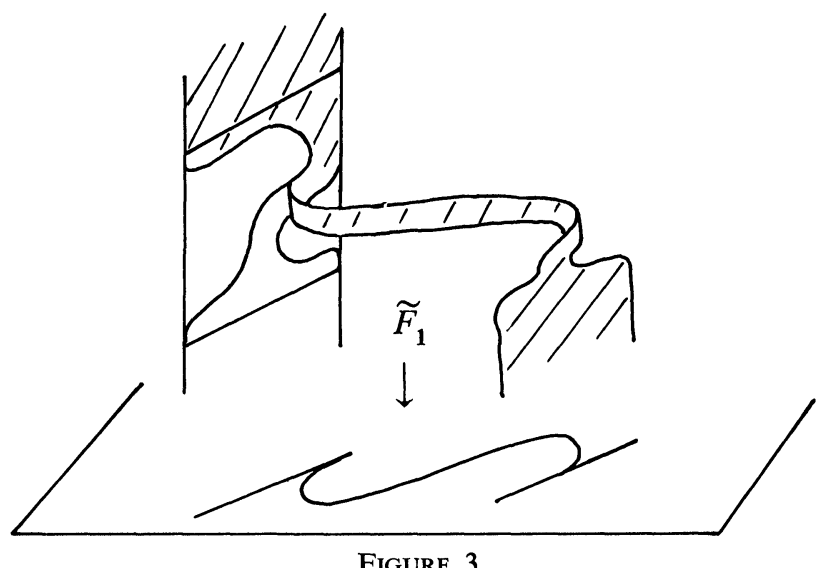

transverse to the foliation defined by $F$; then $F \circ i^{\prime}$ is also an immersion. Next, since $\lambda \leqq a(n)$ this immersion can be $C^{1}$-approximated by an embedding $i^{\prime \prime}$. If the approximation is good enough, $F \circ i^{\prime \prime}$ will still be an immersion. Finally we use $\lambda \leqq a(n)$ to conclude that $i$ and $i^{\prime \prime}$ are isotopic.

3. Theorem $\mathbf{1}^{\prime}$ implies Theorem 1. Pick $l$ sufficiently large so that geo $\operatorname{dim} M \leqq a(n+l)$, and let $M^{\prime}=M \times R^{l}$. This manifold satisfies the hypothesis of Theorem $1^{\prime}$. Give $M$ a metric and $M^{\prime}$ the product metric. Let $p: M^{\prime} \rightarrow M$ be the projection and $i: M \rightarrow M^{\prime}$ the inclusion as $M \times\{0\}$.

I will prove that $d$ induces a bijection of connected components, i.e. that $d_{*}: \pi_{0} \operatorname{Hom}_{k}(M, W) \cong \pi_{0} \operatorname{Lin}_{k}(T M, T W)$. Higher homotopy groups can be treated analogously.

(a) $d_{*}$ is onto. Given $H \in \operatorname{Lin}_{k}(T M, T W)$, the composition $H^{\prime}=$ $H \circ d p: T M^{\prime} \rightarrow T W$ is homotopic to $d F$, for some $F \in \operatorname{Hom}_{k}\left(M^{\prime}, W\right)$, by Theorem $1^{\prime}$. The projection $T M \rightarrow \operatorname{ker} H^{\perp}=\left(\operatorname{ker} H^{\prime}\right)^{\perp} \mid M$ is therefore homotopic to an epimorphism $T M \rightarrow \operatorname{ker} d F^{\perp}$ covering $i$. It follows (see $[3,4.4 .1]$, and [6]) that $i$ is homotopic to a smooth map $\varphi: M \rightarrow M^{\prime}$ transverse to $\operatorname{ker} d F$, and that $H$ is homotopic to $d(F \circ \varphi)$, the differential of a map of rank $k$.

(b) $d_{*}$ is one-one. Suppose given $f, g \in \operatorname{Hom}_{k}(M, W)$ and a homotopy $G_{t}$ in $\operatorname{Lin}_{k}(T M, T W)$ joining $d f$ to $d g$. Composing with $d p$ gives an arc $G_{t}^{\prime}$ joining $d(f \circ p)$ to $d(g \circ p)$; by Theorem $1^{\prime}$ the arc $G_{t}^{\prime}$ is homotopic with fixed endpoints to an arc $d F_{t}$, with $F_{0}=f \circ p, F_{1}=g \circ p$. It follows that the arc of projections $T M \rightarrow \operatorname{ker} G_{t}^{\perp}=\left(\operatorname{ker} G_{t}^{\prime}\right)^{\perp} \mid M$ is homotopic to an arc of epimorphisms $T M \rightarrow \operatorname{ker} d F_{t}^{\perp} \mid M$, which we consider as an arc $H_{t}$ of maps $T M \rightarrow T M^{\prime}$, with $H_{t}$ transverse to ker $F_{t}$. Assertion. This arc is homotopic through such arcs to the arc of the differentials of an arc $\varphi_{t}: M \rightarrow M^{\prime}$ with $\varphi_{t}$ transverse to ker $F_{t}$. We return to this assertion in a 
moment. It is easy to check, using [3] or [6] again, that $i$ is homotopic to $\varphi_{0}$ through maps transverse to $\operatorname{ker} d F_{0}$, and homotopic to $\varphi_{1}$ through maps transverse to $\operatorname{ker} d F_{1}$, so that a homotopy in $\operatorname{Hom}_{k}(M, W)$ between $f$ and $g$ may be described by

$$
f=F_{0} \circ i \sim F_{0} \circ \varphi_{0} \sim F_{1} \circ \varphi_{1} \sim F_{1} \circ i=g .
$$

The assertion is an application of [3]. Let

$$
A(M)=\left\{H \in \operatorname{Lin}\left(T M, T M^{\prime}\right)^{I} \mid H_{t} \text { is transverse to ker } d F_{t} \text { for } t \in I\right\}
$$

and $B(M)=\left\{f \in \operatorname{Hom}\left(M, M^{\prime}\right)^{I} \mid d \circ f \in A(M)\right\}$. Here $\operatorname{Hom}\left(M, M^{\prime}\right)$ is the space of smooth maps: $M \rightarrow M^{\prime}$ with the $C^{1}$-compact-open topology, $\operatorname{Lin}\left(T M, T M^{\prime}\right)$ is the space of continuous, fiberwise linear maps: $T M \rightarrow$ $T M^{\prime}$ with the compact-open topology, and $X^{Y}$ is the space of continuous maps: $Y \rightarrow X$, with the compact-open topology. It follows from [3, 2.4.1, Corollary to 3.2 .3 and 3.4.1] that the "differential" $d: B(M) \rightarrow A(M)$ is a w.h.e.

\section{REFERENCES}

1. A. Dold, Partitions of unity in the theory of fibrations, Ann. of Math. (2) 78 (1963), 223-255. MR 27 \#5264.

2. S. Feit, $k$-mersions of manifolds, Acta Math. 122 (1969), 173-195. MR 39 \#4862.

3. M. L. Gromov, Stable mappings of foliations into manifolds, Izv. Akad. Nauk SSSR 33 (1969), 707-734 = Math. USSR Izv. 3 (1969), 671-694. MR 41 \#7708.

4. A. Haefliger, Feuilletages sur les variétés ouvertes, Topology 9 (1970), 183-194. MR 41 \#7709.

5. M. Hirsch, Immersions of manifolds, Trans. Amer. Math. Soc. 93 (1959), 242-276. MR 22 \#9980.

6. A. Phillips, Smooth maps transverse to a foliation, Bull. Amer. Math. Soc. 76 (1970), 792-797. MR 41 \#7711.

7. - Submersions of open manifolds, Topology 6 (1967), 171-206. MR 34 \#8420.

8. S. Smale, The classification of immersions of spheres in Euclidean spaces, Ann. of Math. (2) 69 (1959), 327-344. MR 21 \#3862.

Department of Mathematics, State University of New York, Stony Brook, NEW YORK 11790 